\begin{tabular}{|c|c|}
\hline Title & Optical generation of surface acoustic waves guided at the linear boundary between two thin films \\
\hline Author(s) & Maznev, A . A .; Kelf, Timothy A .; Tomoda, Motonobu; Matsuda, O samu; Wright, Oliver B. \\
\hline Citation & $\begin{array}{l}\text { Journal of A pplied Physics, 107(3), } 033521 \\
\text { https://doi.org/10.1063/1.3298472 }\end{array}$ \\
\hline Issue Date & 2010-02-01 \\
\hline Doc URL & http:/hdl. handle.net/2115/42696 \\
\hline Rights & $\begin{array}{l}\text { Copyright } 2010 \text { A merican Institute of Phy sics. This article may be downloaded for personal use only. A ny other use } \\
\text { requires prior permission of the author and the A merican Institute of Physics. The following article appeared in J. A ppl. } \\
\text { Phys. 107, } 033521 \text { (2010) and may be found at https://dx.doi.org } 10.1063 / 1.3298472\end{array}$ \\
\hline Type & article \\
\hline File Information & JAP107-3_033521.pdf \\
\hline
\end{tabular}

Instructions for use 


\title{
Optical generation of surface acoustic waves guided at the linear boundary between two thin films
}

\author{
A. A. Maznev, ${ }^{1,2, a)}$ Timothy A. Kelf, ${ }^{1}$ Motonobu Tomoda, ${ }^{1}$ Osamu Matsuda, ${ }^{1}$ and \\ Oliver B. Wright ${ }^{1}$ \\ ${ }^{1}$ Division of Applied Physics, Graduate School of Engineering, Hokkaido University, Sapporo 060-8628, \\ Japan \\ ${ }^{2}$ Institute of Physical Chemistry, University of Heidelberg, Im Neuenheimer Feld 253, D-69120 Heidelberg, \\ Germany
}

(Received 27 October 2009; accepted 2 January 2010; published online 9 February 2010)

\begin{abstract}
Laser-induced transient grating measurements and ultrafast optical pump-probe imaging of surface acoustic waves near a linear boundary between copper and silica films on a silicon substrate indicate the presence of a boundary-localized mode with a phase velocity slightly below the Rayleigh wave velocity on the copper film. We analyze in detail the dispersion of this localized mode in comparison with that of the Rayleigh waves in the surrounding materials. The existence of the localized mode is ascribed to the nonuniformity of the copper film thickness near its edge resulting from polishing during fabrication. (c) 2010 American Institute of Physics. [doi:10.1063/1.3298472]
\end{abstract}

\section{INTRODUCTION}

Extensive research on laser-generated surface acoustic waves (SAWs) in supported thin films ${ }^{1,2}$ has been primarily motivated by film thickness and mechanical property measurements. Initially this work largely involved "blanket" thin films that for all intents and purposes can be considered infinite. Recently, laser-generated SAWs in patterned thin film microstructures comprised of different materials have also attracted attention. $^{3-6}$ This activity is stimulated by the proliferation of photoacoustic techniques in process control applications in silicon microelectronics, ${ }^{7,8}$ where patterned thin film structures play a crucial role.

The interaction of SAWs with the linear boundary between two thin films made of different materials on a substrate is an interesting but little-studied problem. SAWs should undergo refraction and reflection at such a boundary ${ }^{9,10}$ in a similar way to bulk acoustic waves at a planar interface. ${ }^{11}$ It is known that under certain conditions a planar interface between two solids may support a guided acoustic mode referred to as a Stoneley wave. ${ }^{11-13}$ An interfacial wave may also exist if one of the media is inhomogeneous near the interface. ${ }^{14} \mathrm{~A}$ surface wave localized at a linear interface between two thin films would present a surface analog of the Stoneley wave. Guided waves of this kind have been studied neither theoretically nor experimentally. The possibility of the existence of surface waves localized at a boundary between two "quarter spaces" filled with different materials has been suggested, ${ }^{15}$ but no evidence that such waves actually exist has been reported.

In this study, we present experimental observations of a surface acoustic mode guided at a linear boundary between two thin film structures on a silicon substrate. We believe that the observed guided wave is associated with a waveguide channel near the boundary formed due to a film thickness nonuniformity, and thus does not present a true analog

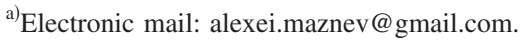

of an interface wave between two homogeneous materials. The question of the existence of surface waves guided at a linear boundary between two uniform thin films remains open, and we hope that this work will stimulate the search for such waves. The present study also involves a practical aspect: laser-generated SAWs are routinely used for metal film thickness profiling, ${ }^{8}$ and the existence of a localized boundary mode significantly affects measurements performed close to the boundary.

\section{EXPERIMENT}

\section{A. Sample}

The sample manufactured by International Sematech comprises various copper test structures embedded in silicon oxide $\left(\mathrm{SiO}_{2}\right)$ on a $200 \mathrm{~mm}$ (100) silicon wafer. One of the structures is a $0.5 \times 0.5 \mathrm{~mm}^{2}$ copper square surrounded by the oxide film, as shown in Fig. 1(a), with the sides of the square aligned along the [110] direction of $\mathrm{Si}$. A cross section of the sample is schematically shown in Fig. 1(b). The patterned copper structure is fabricated by the so-called damascene process, ${ }^{16}$ which involves patterning the dielectric film, depositing a Ta diffusion barrier and a seed $\mathrm{Cu}$ layer by physical vapor deposition, depositing the bulk of $\mathrm{Cu}$ by electroplating, and, finally, polishing off the excess copper by chemical-mechanical polishing (CMP). Ideally, the $\mathrm{Cu}$ surface should be level with the surrounding dielectric. Often in practice and in the case of our sample, however, the $\mathrm{Cu}$ is overpolished and recessed with respect to the surface of the dielectric. This phenomenon called "dishing" is especially pronounced on the larger $\mathrm{Cu}$ pads on the wafer. Figures 2(a) and 2(b) show atomic force microscope (AFM) profiles measured across the $\mathrm{Cu} / \mathrm{SiO}_{2}$ boundary on two identical structures respectively located at the center and at the edge of the wafer. Copper dishing on our wafer is significant, especially at the wafer edge where it is about twice as large $(160 \mathrm{~nm})$ as at the wafer center $(80 \mathrm{~nm})$. 
(a)

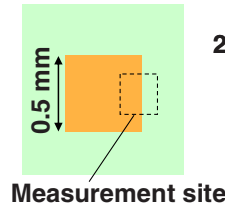

(b)

(c)

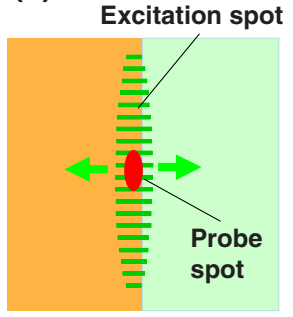

(d)
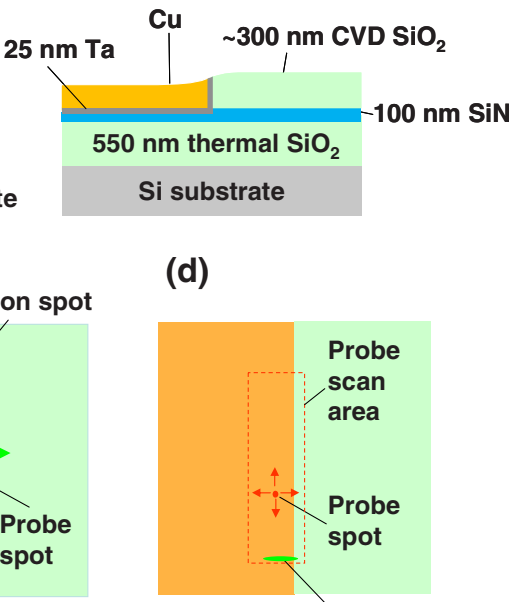

Excitation spot

FIG. 1. (Color online) Schematic diagrams of the sample. (a) Top view of the copper pad. (b) Cross section of the sample near the edge of the pad (not to scale). (c) Measurement site for the laser-induced grating experiment with the pump and probe laser spots schematically shown; both spots are scanned in the horizontal direction. (d) Measurement site for the ultrafast imaging experiment with the pump and probe laser spots schematically shown; the pump spot position is fixed while the probe spot is scanned in both directions.

\section{B. Transient grating apparatus}

The transient grating setup, involving optical heterodyne detection, has been described in detail previously. ${ }^{17}$ In summary, two excitation pulses derived from a single laser (of pulse duration $0.5 \mathrm{~ns}$, wavelength $532 \mathrm{~nm}$, total energy at the sample $\sim 1 \mu \mathrm{J}$, and repetition rate $1.5 \mathrm{kHz}$ ) are crossed at the sample surface to form sinusoidal fringes with a period in the range 4-10 $\mu \mathrm{m}$. Absorption of the excitation light followed by rapid thermal expansion generates counterpropagating acoustic modes at a wavevector defined by the periodicity of the excitation grating. Detection of the surface ripples associated with surface acoustic modes is performed by means of the diffraction of the quasi-cw probe beam (of wavelength $830 \mathrm{~nm}$, power at the sample $\sim 100 \mathrm{~mW}$ ) focused at the center of the excitation pattern. The diffracted beam, amplified by the use of optical heterodyning, is detected with a fast photodiode and fed to a digital oscillo-

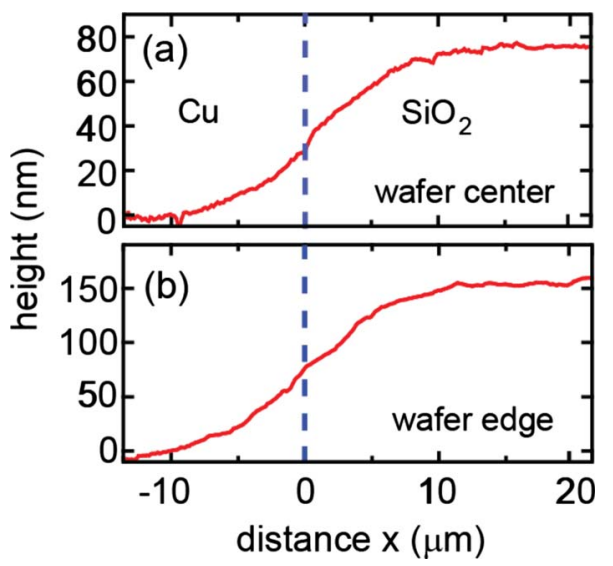

FIG. 2. (Color online) AFM profiles across the $\mathrm{Cu} / \mathrm{SiO}_{2}$ boundary (dashed line) measured (a) at the wafer center and (b) at the wafer edge. scope, yielding an effective detection bandwidth $\sim 1 \mathrm{GHz}$. The excitation spot size is $300 \times 50 \mu \mathrm{m}^{2}$ (with the long dimension along the wavevector of the grating), and the probe spot size is $50 \times 25 \mu \mathrm{m}^{2}$.

As shown in Fig. 1(c), the excitation pattern is aligned to produce an acoustic wavevector directed along the boundary between the $\mathrm{Cu}$ and $\mathrm{SiO}_{2}$ films. The sample is put on a motorized stage and translated perpendicular to the boundary.

\section{Ultrafast pump-probe imaging setup}

For surface wave imaging we use an optical pump and probe technique combined with a common-path Sagnac interferometer. ${ }^{18}$ We use normally-incident subpicosecondduration $400 \mathrm{~nm}, 0.3 \mathrm{~nJ}$ pump pulses from a Ti:sapphire laser at a repetition rate of $75.8 \mathrm{MHz}$ to thermoelastically excite broadband SAWs. These pulses are focused with a combination of a cylindrical lens and a $\times 50$ microscope objective to an elongated elliptical spot of dimensions $30 \times 2 \mu \mathrm{m}^{2}$ on a point on the copper situated adjacent to the $\mathrm{Cu} / \mathrm{SiO}_{2}$ film boundary, as shown in Fig. 1(d). SAW frequencies up to $\sim 1 \mathrm{GHz}$ are generated, propagating parallel to the boundary, with the highest acoustic amplitudes occurring around $400 \mathrm{MHz}$ (or a SAW wavelength of $\lambda \sim 10 \mu \mathrm{m}$ ). The pump beam is chopped at $1 \mathrm{MHz}$ for synchronous lock-in detection. The probe beam is focused through the same objective lens to an approximately circular spot of diameter $2 \mu \mathrm{m}$. We use two delayed $800 \mathrm{~nm}$ optical probe pulses separated in time by 330 ps to detect an optical phase difference proportional to the out-of-plane velocity of the sample surface. A scanning system based on tilting mirrors raster scans the probe spot in two dimensions relative to the pump spot. The pump-probe delay time is incremented after each such spatial image has been acquired in order to obtain a series of 64 images over the $13.2 \mathrm{~ns}$ period of the laser pulse repetition.

\section{RESULTS AND DISCUSSION}

\section{A. Transient grating measurements}

Figure 3(a) shows the detected signal at an acoustic wavelength of $5 \mu \mathrm{m}$ measured on the $\mathrm{Cu}$ pad at the wafer center at a distance of $50 \mu \mathrm{m}$ from the edge of the pad. The sharp rise from the zero level corresponds to the moment of sample excitation. The high frequency oscillations are due to SAWs, whereas the slowly decaying component is due to the contribution of the "thermal grating" associated with the periodic temperature profile. ${ }^{2}$ The spectrum of the acoustic oscillations shown in the inset reveals two acoustic modes of the film/substrate system, referred to as Rayleigh and Sezawa waves, ${ }^{11,12}$ the latter being much weaker in amplitude. The measured phase velocity dispersion for the two modes is presented in Fig. 3(b). Using a numerical analysis, ${ }^{2,19}$ dispersion curves were fitted to determine the thickness of $\mathrm{Cu}$ and $\mathrm{SiN}$, as shown by the solid lines in Fig. 3(b). The SiN layer had an initial thickness of $100 \mathrm{~nm}$, and served as an etch stop in the damascene process; it was partially etched away prior to the $\mathrm{Cu}$ deposition. The fitting procedure resulted in good agreement between the measured and calculated dispersion curves, and yielded thicknesses $229 \mathrm{~nm} / 42 \mathrm{~nm}$ for $\mathrm{Cu} / \mathrm{SiN}$, respectively at the wafer center and $148 \mathrm{~nm} / 33 \mathrm{~nm}$ at the 

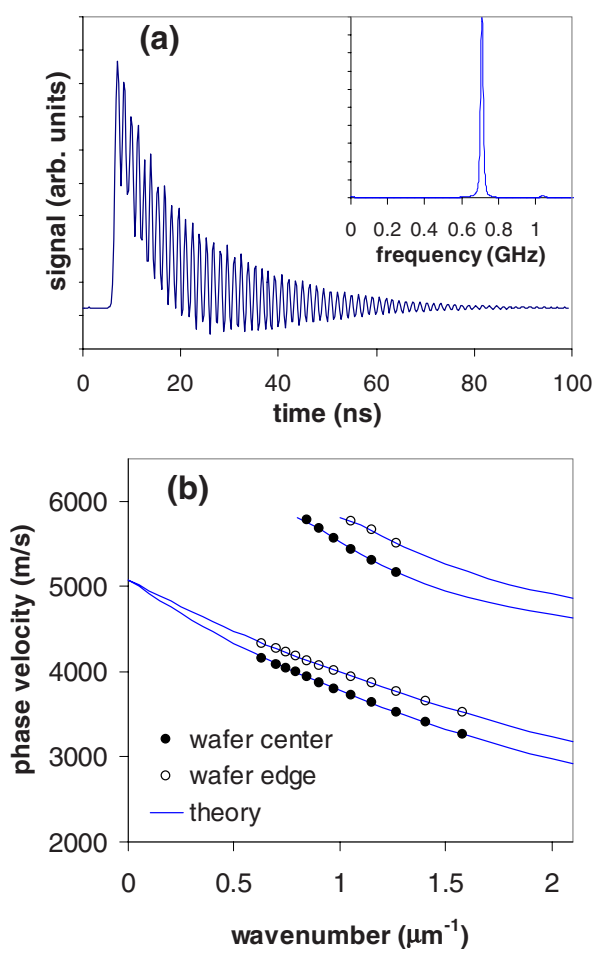

FIG. 3. (Color online) (a) Signal waveform from the laser-induced grating experiment and Fourier spectrum of the acoustic oscillations (inset) inside the copper pad at a point $50 \mu \mathrm{m}$ from the boundary at the acoustic wavelength $5 \mu \mathrm{m}$, measured at the wafer center. (b) Measured (circles) and calculated (solid lines) dispersion curves of surface acoustic modes for the copper pad at a point $50 \mu \mathrm{m}$ from the boundary. Solid circles correspond to measurements at the wafer center, open circles to measurements at the wafer edge.

wafer edge. The difference in the $\mathrm{Cu}$ thickness resulting from the overpolishing is in agreement with the AFM profiles.

Figures 4 and 5 show what happens when the laser spot is scanned across the boundary between copper and silica. As
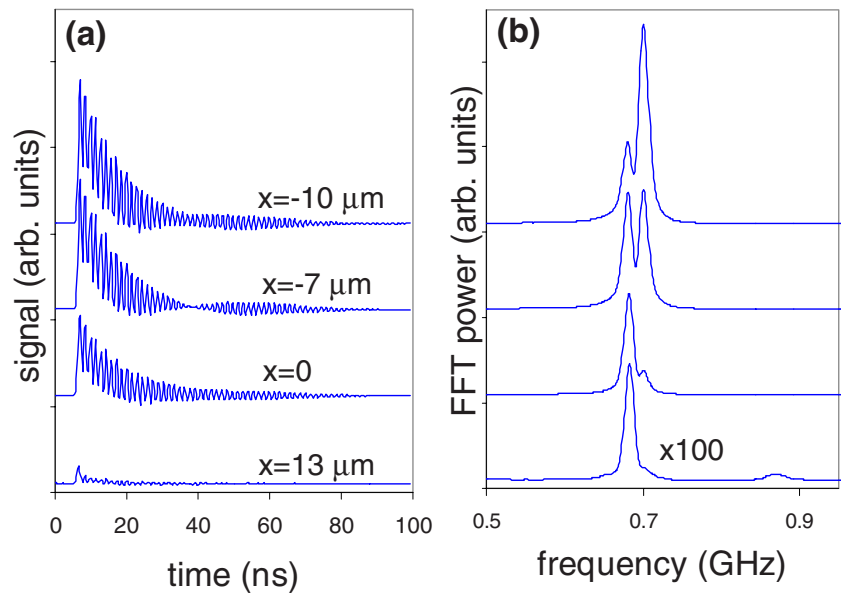

FIG. 4. (Color online) (a) Signal waveforms from the laser-induced grating experiment, and (b) corresponding spectra, at different distances of the center of the probe laser spot from the boundary at the acoustic wavelength $5 \mu \mathrm{m}$, measured at the wafer center. The negative sign corresponds to the position to the left of the boundary, i.e., inside the copper pad. The distance is controlled with $\sim 1 \mu \mathrm{m}$ precision, small compared to the laser spot size. The intensity of the spectrum shown at the bottom was increased by a factor of 100 to make it comparable in amplitude with other spectra. The smal high frequency peak in the bottom spectrum corresponds to the Rayleigh wave in the $\mathrm{SiO}_{2}$ field.

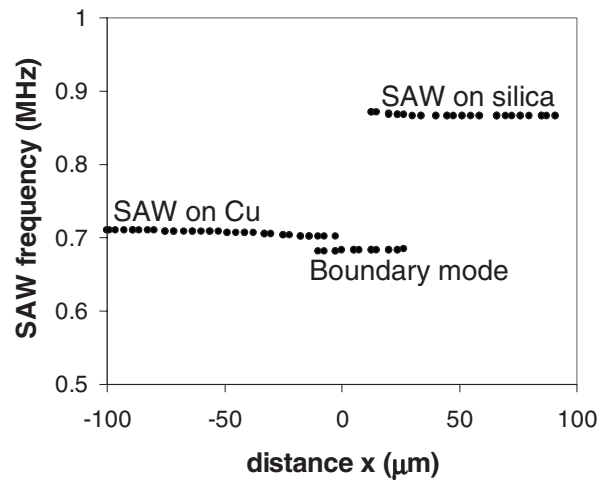

FIG. 5. The dependence of the acoustic mode frequencies at the acoustic wavelength $5 \mu \mathrm{m}$ on the laser spot position with respect to the boundary, measured with the laser-induced grating technique at the wafer center.

expected, outside the copper pad the Rayleigh wave frequency is significantly higher than inside the pad owing to the higher acoustic velocities and lower density of $\mathrm{SiO}_{2}$ compared to $\mathrm{Cu}$. The signal inside the copper pad is much higher, as both the generation and detection of SAWs are more efficient due to the higher expansion coefficient of $\mathrm{Cu}$ and due to its higher reflectivity for the probe beam. In the proximity of the boundary, a new peak, slightly lower in frequency than the Rayleigh wave on $\mathrm{Cu}$, emerges in the spectrum. This peak apparently corresponds to a waveguide mode localized near the boundary, whose frequency and, hence, phase velocity is lower compared to SAWs.

Figures 6(a) and 6(b) show the dispersion curves for this localized mode together with that of the Rayleigh wave, measured at the center and the edge of the wafer, respectively. One can see that the separation between the modes at
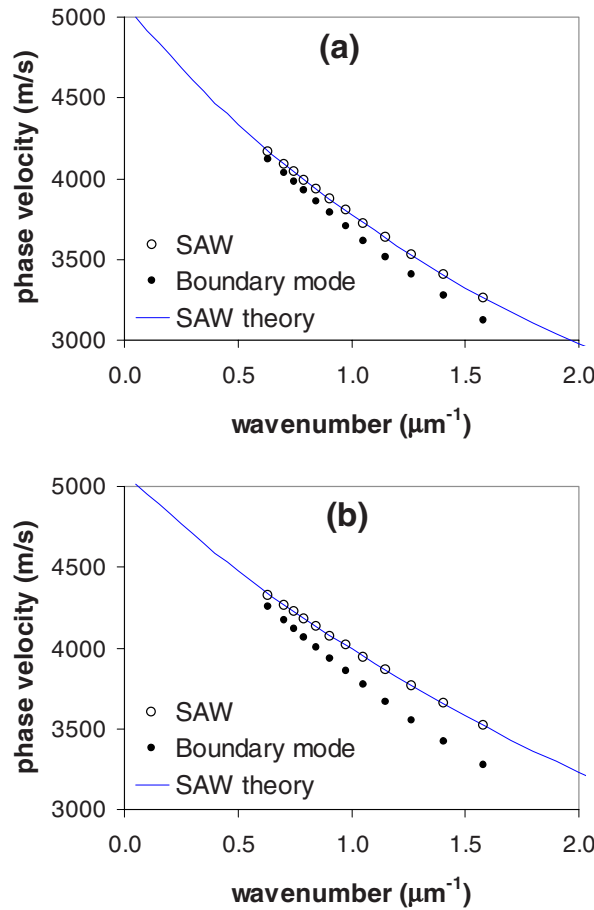

FIG. 6. (Color online) Dispersion curves of the boundary mode (closed circles) and the Rayleigh waves on $\mathrm{Cu}$ (open circles: measurements, solid lines: calculations) measured at (a) the wafer center and (b) the wafer edge with the laser-induced grating technique. 

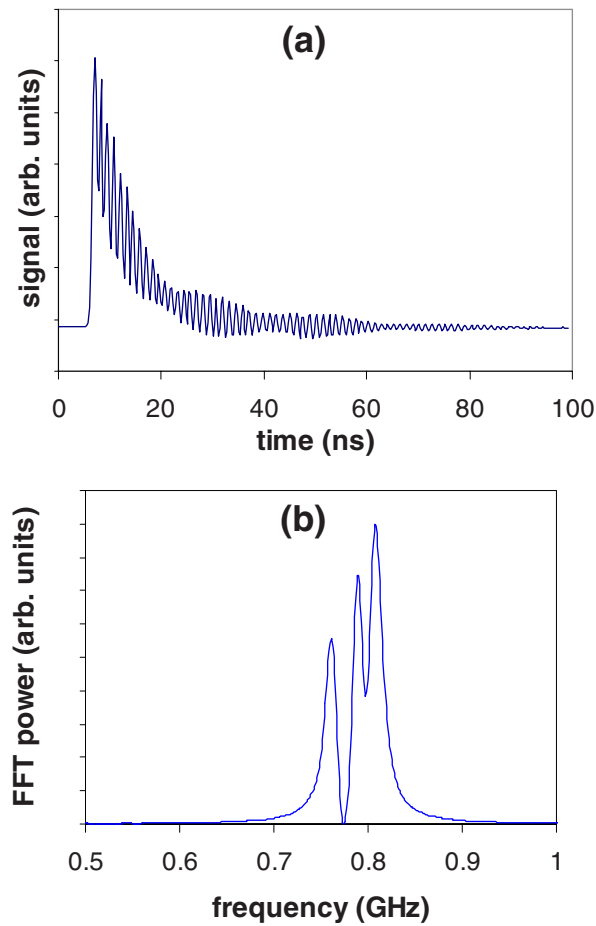

FIG. 7. (Color online) (a) Laser-induced grating signal waveform and (b) spectrum measured at the wafer edge at the acoustic wavelength $4.5 \mu \mathrm{m}$ (wavenumber $1.41 \mathrm{~mm}^{-1}$ ), with the center of the laser spot located inside the copper pad at a distance of $7 \mu \mathrm{m}$ from the boundary.

the wafer edge is about twice as large as at the center. This fact suggests that the dishing of copper, which is about twice as large at the wafer edge as at the center, is the key factor responsible for the existence of the boundary mode. Thicker $\mathrm{Cu}$ yields a lower SAW velocity, and thus the strip of thicker copper near the boundary of the pad forms a waveguide channel for SAWs. For the structure located at the wafer edge, another guided mode splits from the Rayleigh wave peak at short wavelengths, as can be seen in Fig. 7. The appearance of higher order modes at shorter wavelengths is another typical characteristic of a waveguide channel.

\section{B. Ultrafast imaging measurements}

The transient grating technique yields accurate frequency measurements of the localized boundary mode, but the information obtained from these measurements is spatially averaged owing to the size of the probe spot. To investigate the acoustic field in the boundary mode, we turn to the ultrafast pump-probe imaging technique. A real time movie of a $50 \times 250 \mu \mathrm{m}^{2}$ region at the edge of the $\mathrm{Cu}$ pad at the wafer center is available in the supplementary information. ${ }^{20}$ The real time movie is comprised of a superposition of SAWs at the excited frequencies, corresponding to multiples of the laser repetition rate. Single frequency images were obtained by temporal Fourier transforms. ${ }^{21}$ Figures 8(a) and 8(b) show images corresponding to 531 and $455 \mathrm{MHz}$, respectively. The elliptical pump spot is located at the bottom of these images, as indicated in the figures. In each case one can clearly identify the boundary mode localized at the edge of the copper pad having a smaller wavelength (and, consequently, a smaller phase velocity) than the Rayleigh wave

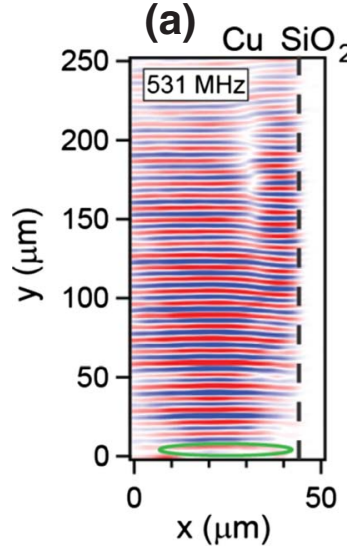

(b)

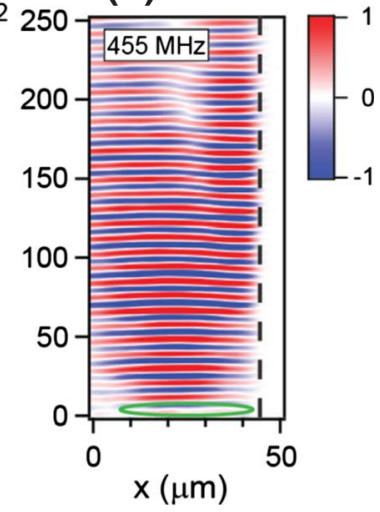

(c)

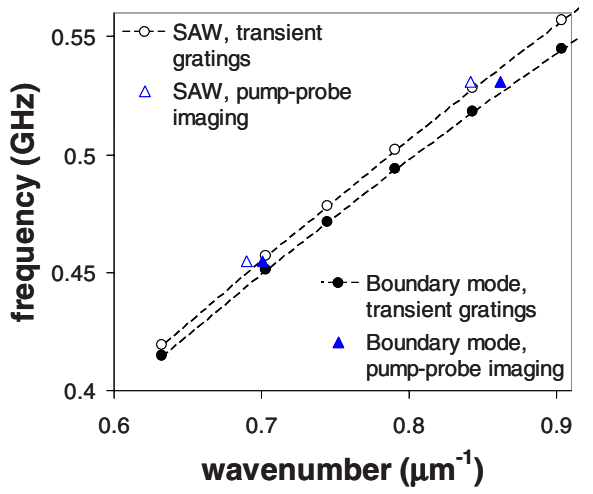

FIG. 8. (Color online) Frequency-filtered images of the acoustic displacement at (a) $531 \mathrm{MHz}$ and (b) $455 \mathrm{MHz}$ measured by the ultrafast imaging technique at the wafer center. The dashed lines show the $\mathrm{Cu} / \mathrm{SiO}_{2}$ boundary. The ellipses at the bottom of the images show the source position. (c) Comparison of the wavenumber vs frequency measurements based on the images in (a) and (b) (shown by triangles) with the frequency vs wavenumber measurements done with the transient grating technique (shown by circles). Open symbols correspond to the Rayleigh wave, closed symbols to the boundary mode. Dashed lines connecting the points measured by the transient grating method serve as a guide to the eye.

inside the $\mathrm{Cu}$ pad. Figure 8(c) presents a comparison of the acoustic wave numbers measured from the images as a function of frequency with the results of the transient grating measurements. There is a difference in the method of data acquisition in our two experimental techniques. In the ultrafast imaging method, we measure the wave number as a function of frequency, whereas in the transient grating method we measure the frequency as a function of the wave number. Figure $8(\mathrm{c})$ indicates a small $(\sim 1 \%)$ systematic discrepancy between the two techniques. This error could arise from a combination of a small difference in measurement positions on the $\mathrm{Cu}$ pad or from a small discrepancy in the calibration of the distance scales in the optical techniques.

Because the velocity of the guided boundary mode is very close to that of the Rayleigh wave, the former is not entirely localized within the waveguide. This can be clearly seen in Fig. 8(b), where this guided boundary mode occupies a strip more than $10 \mu \mathrm{m}$ in width, whereas the width of the region with a higher $\mathrm{Cu}$ thickness, according to the AFM profiles shown in Fig. 2, is less than $10 \mu \mathrm{m}$. Indeed, outside the waveguide, that is in the area where the film thickness is uniform, the amplitude $U$ of a waveguide mode should undergo exponential decay ${ }^{22}$ 


$$
U \propto \exp \left(-k_{x}|x|\right)
$$

where $x$ is the coordinate perpendicular to the waveguide, and

$$
k_{x}=k \sqrt{1-\frac{v^{2}}{v_{R}^{2}}},
$$

where $k$ and $v$ are the wavenumber and phase velocity of the waveguide mode, respectively, and $v_{R}$ is the phase velocity of the Rayleigh wave outside of the waveguide. If $v$ is close to $v_{R}$, we obtain the following result for the penetration depth $\gamma$ outside the waveguide:

$$
\gamma=1 / k_{x}=\frac{\lambda}{2^{3 / 2} \pi} \sqrt{\frac{v_{R}}{\left(v_{R}-v\right)}},
$$

where $\lambda$ is the wavelength of the guided mode. The waveguide mode should therefore become more delocalized at long wavelengths as the velocity difference diminishes. In our case, the relative velocity difference between the guided boundary mode and the SAW within the $\mathrm{Cu}$ pad is $2.3 \%$ at $531 \mathrm{MHz}$ and $1.5 \%$ at $455 \mathrm{MHz}$. Accordingly, Eq. (3) gives a penetration depth into the $\mathrm{Cu}$ pad outside the waveguide equal to $\gamma=5.5 \mu \mathrm{m}$ in the former case and $\gamma=8.5 \mu \mathrm{m}$ in the latter case. The increase in the value of $\gamma$ with decreasing frequency qualitatively accounts for the increase in the width of the strip evident in Figs. 8(a) and 8(b) that is affected by the boundary mode. The penetration depths into the area outside the copper pad, according to Eq. (2), are $\gamma=2$ and $2.8 \mu \mathrm{m}$ at 531 and $455 \mathrm{MHz}$, respectively. These much smaller penetration depths arise because of the significantly larger Rayleigh wave velocity outside the pad. Unfortunately, the low signal strength outside the $\mathrm{Cu}$ pad did not allow an accurate measurement of the acoustic field distribution there.

\section{Discussion}

Our measurements indicate that the observed boundary mode owes its existence to the waveguide channel formed by the copper film thickness nonuniformity near the boundary. It is not unexpected that such a film thickness variation results in a SAW waveguide. Thin film waveguides formed by depositing a strip of a "slow" material on a substrate are well known. ${ }^{11,22}$ In our case, the waveguide is formed at the boundary of two films, which makes the analysis more complex. This problem should therefore present an interesting subject for numerical simulations which may also help answer the question of whether a guided boundary mode may exist in the absence of the film thickness nonuniformity.

Measurement of the thickness of metal films by lasergenerated SAWs is performed by commercially available instruments at microelectronics factories. Knowing the existence of boundary modes is important for the correct interpretation of such measurements. For example, if one wished to measure a copper thickness profile on the sample used in this study based on the frequency of the largest peak in the spectrum, it would appear that the thickness undergoes a sharp jump near the boundary. However, in reality the thickness profile is smooth. On the other hand, since the boundary mode results from the dishing of copper, it might prove useful in CMP process control: for example, the presence or absence of the boundary mode at certain wavelengths may indicate that the dishing is above/below acceptable levels.

\section{CONCLUSIONS}

By analogy to the way in which bulk acoustic waves may become guided by an interface between two media, SAWs can become localized at a linear edge or a boundary between two thin film structures. Such guided modes are decoupled from surface waves in the areas on either side of the boundary as well as from the bulk waves in the substrate, and can only be excited by a source located in close proximity to the boundary. Laser acoustics thus presents a very convenient tool for studying these modes. We have demonstrated that dispersion of the localized boundary modes can be measured with laser-induced transient gratings, whereas their spatial distribution can be measured with an ultrafast imaging technique.

The boundary mode reported in this paper is guided by a region of thicker copper near the edge of the film. The question of whether there exists a one-dimensional analog of the Stoneley wave, that is a localized surface mode guided by a linear boundary between two films of uniform thickness on a substrate, or between two media each occupying a quarter space, is still open. The experimental techniques used in this work present an adequate tool for looking for such waves.

\section{ACKNOWLEDGMENTS}

Donation of the measurement system by Advanced Metrology Systems, L. L. C. is greatly appreciated. The contribution by A.A.M. was supported in part by the Alexander von Humboldt Foundation.

${ }^{1}$ A. Neubrand and P. Hess, J. Appl. Phys. 71, 227 (1992).

${ }^{2}$ J. A. Rogers, A. A. Maznev, M. J. Banet, and K. A. Nelson, Annu. Rev. Mater. Sci. 30, 117 (2000).

${ }^{3}$ D. M. Profunser, O. Matsuda, and O. B. Wright, Phys. Rev. Lett. 97, 055502 (2006).

${ }^{4}$ C. Giannetti, B. Revaz, F. Banfi, M. Montagnese, G. Ferrini, F. Cilento, S. Maccalli, P. Vavassori, G. Oliviero, E. Bontempi, L. E. Depero, V. Metlushko, and F. Parmigiani, Phys. Rev. B 76, 125413 (2007).

${ }^{5}$ J.-F. Robillard, A. Devos, I. Roch-Jeune, and P. A. Mante, Phys. Rev. B 78, 064302 (2008).

${ }^{6}$ A. A. Maznev, Ultrasonics 49, 1 (2009).

${ }^{7}$ C. Diebold and R. Stoner, Handbook of Silicon Semiconductor Metrology, edited by A. C. Diebold (Dekker, New York, 2001), p. 197.

${ }^{8}$ M. Gostein, M. Banet, M. Joffe, A. A. Maznev, R. Sacco, J. Rogers, and K. A. Nelson, Handbook of Silicon Semiconductor Metrology, edited by A. C. Diebold (Dekker, New York, 2001), p. 167.

${ }^{9} \mathrm{P}$. Malischewsky, Surface Waves and Discontinuities (Elsevier, Amsterdam, 1987)

${ }^{10}$ D. H. Hurley, O. B. Wright, O. Matsuda, T. Suzuki, S. Tamura, and Y. Sugawara, Phys. Rev. B 73, 125403 (2006).

${ }^{11}$ B. A. Auld, Acoustic Fields and Waves in Solids, 2nd ed. (Krieger, Malabar, 1990), Vol. II.

${ }^{12}$ G. W. Farnell and E. L. Adler, in Physical Acoustics, Principles and Methods, edited by W. P. Mason and R. N. Thurston (Academic, New York, 1972), Vol. 9, p. 35.

${ }^{13}$ R. O. Claus and C. H. Palmer, Appl. Phys. Lett. 31, 547 (1977).

${ }^{14}$ O. A. Godin and D. M. F. Chapman, J. Acoust. Soc. Am. 110, 1890 (2001).

${ }^{15}$ A. P. Mayer, Ultrasonics 48, 478 (2008). 
${ }^{16}$ R. Rosenberg, D. C. Edelstein, C.-K. Hu, and K. P. Rodbell, Annu. Rev. Mater. Sci. 30, 229 (2000)

${ }^{17}$ A. A. Maznev, A. Mazurenko, L. Zhuoyun, and M. Gostein, Rev. Sci. Instrum. 74, 667 (2003).

${ }^{18}$ T. Tachizaki, T. Muroya, O. Matsuda, Y. Sugawara, D. H. Hurley, and O. B. Wright, Rev. Sci. Instrum. 77, 043713 (2006).

${ }^{19}$ The following values of density and elastic constants of the constituent materials were used in the calculations: $\mathrm{Cu}: \rho=8930 \mathrm{~kg} / \mathrm{m}^{3}, \quad E$ $=128.7 \mathrm{GPa}, \nu=0.344 ; \mathrm{Ta}: \rho=16654 \mathrm{~kg} / \mathrm{m}^{3}, E=184.2 \mathrm{GPa}, \nu=0.342$; $\mathrm{SiN}: \rho=2650 \mathrm{~kg} / \mathrm{m}^{3}, E=171.7 \mathrm{GPa}, \nu=0.161 ; \mathrm{SiO}_{2}: \rho=2200 \mathrm{~kg} / \mathrm{m}^{3}, E$ $=71.3 \mathrm{GPa}, \quad \nu=0.164 ; \quad \mathrm{Si}: \rho=2330 \mathrm{~kg} / \mathrm{m}^{3}, \quad C_{11}=165.7 \mathrm{GPa}, \quad C_{12}$
$=63.7 \mathrm{GPa}, C_{44}=79.6 \mathrm{GPa}$. Thin film materials were modeled as elastically isotropic and their elastic properties are listed above in terms of the Young's modulus $E$ and the Poisson's ratio $\nu$, whereas for the single crystal silicon substrate three independent terms of the elastic constant tensor are listed.

${ }^{20}$ See EPAPS supplementary material at http://dx.doi.org/10.1063/ 1.3298472 for details on the animation.

${ }^{21}$ D. M. Profunser, E. Muramoto, O. Matsuda, O. B. Wright, and U. Lang, Phys. Rev. B 80, 014301 (2009).

${ }^{22}$ A. A. Oliner, in Acoustic Surface Waves, edited by A. A. Oliner (Springer Verlag, Berlin, 1978), p. 187. 